\title{
Planned Challenge Agent Exposure Rate
}

National Cancer Institute

\section{Source}

National Cancer Institute. Planned Challenge Agent Exposure Rate. NCI Thesaurus. Code C161575.

The planned amount of challenge agent exposure per unit of time. 